\title{
Francisolâ
}

\section{Un dispositif pour les enseignants de FLE des universités angolaises}

\author{
Anselmo ILUNGA \\ Département de français, Universidade Agostinho Neto - Angola \\ Reçu le 2 octobre 2018 | Accepté le 30 décembre 2018
}

\begin{abstract}
RÉSUMÉ. La présente contribution, converge vers la didactique comparée et porte sur la description des dimensions génériques et spécifiques, sur le savoir et les pratiques (Mercier, 2000, Schubauer-Léoni \& Sensevy, 2000) à partir des vidéographies qui nous permettront de décrire les activités de classe en nous intéressant à la fois sur la circulation des savoirs et surtout l'analyse des gestes professionnels des quatre professeurs du FLE de deux institutions universitaires (Groupe $\mathrm{A}$ et groupe B). Le résultat a démontré que les professeurs du groupe $A$ semblent avoir de difficulté par rapport à leur congénère de $B$ qui ont reçu la formation du FLE. Cette analyse didactique nous permettra de trouver des réponses aux difficultés dans l'activité enseignante et permettre aux apprenants d'être des acteurs de leurs propres apprentissages.
\end{abstract}

Mots-clés : approche comparative, FLE, action conjointe, gestes professionnels

\begin{abstract}
This contribution, which converges on comparative didactics, focuses on the description of generic and specific dimensions, knowledge and practices (Mercier, Schubauer-Léoni \& Sensevy, 2000) from the videographies that will allow us to describe the classroom activities by focusing on both the flow of knowledge and, above all, the analysis of the professional gestures of the four FLE professors of two university institutions (Group A and Group B). The result showed that Group A teachers appear to have difficulty with their B congener who received the FLE training. This didactic analysis will allow us to find answers to the difficulties in the teaching activity and allow the learners to be actors of their own learning.
\end{abstract}

Keywords: comparative approach, FLE, joint action, professional gestures

auteur correspondant : anselmoilunga91@gmail.com

Pour citer cet article (Style APA) : Ilunga, A. (2018). Un dispositif pour les enseignants de FLE des universités angolaises. Francisola : Revue Indonésienne de la langue et la littérature françaises, 3(2), 162-175. doi: 10.17509/francisola.v3i2.15749 


\section{INTRODUCTION}

Les nouvelles transformations apportées par la mondialisation et ses corolaires nous mettent en face d'une situation qui fait que l'enseignement se voit obligé d'adopter une certaine ligne de conduite dans la recherche de l'amélioration de ses activités. C'est dans ce cadre que nous avons voulu présenter notre contribution sur les difficultés langagières des professeurs du FLE dans les universités angolaises. Mais nous avons noté que la difficulté des professeurs entraine d'une manière ou d'une autre celle des étudiants et les deux sont liées au contexte.

Les gestes professionnels nous démontrent que la profession enseignante n'est pas si facile qu'on ne le pense. Ce qui suppose une certaine maîtrise des gestes professionnels conséquents qui privilégient certaines postures des étudiants.

Que doit faire le professeur pour améliorer ses pratiques? Que lui faut-il pour l'améliorer?

La recherche porte sur une problématique spécifique qui porte sur les difficultés des étudiants en FLE qui nous interpelle et cela nous amène à préconiser quelques pistes à partir de ces questions : dans quelle mesure les difficultés des étudiants seraient-ils en lien avec les difficultés des enseignants peu formés à enseigner le FLE? Quel est le profil des enseignants et des étudiants ? Quelle formation les enseignants ont-ils reçue? Sur quels programmes officiels s'appuient-ils pour enseigner? Quels dispositifs didactiques et pédagogiques mettent-ils en place pour enseigner? Les gestes professionnels ajustés et une formation transposée de CECRL comme solution pouvant aider les professeurs à améliorer leurs compétences et aux étudiants de mieux s'approprier les postures adéquates.

Notre recherche concerne un milieu multilingue où cohabitent les langues nationales et les langues étrangères.

Nous allons présenter deux approches de la notion des gestes professionnels. La première approche s'appuie sur les auteurs qui conçoivent les gestes en termes de: genre, schème, praxéologie. Il s'agit de G. Sensey (1998), pour qui le geste a pour but de maintenir la relation didactique entre l'enseignant, les apprenants et le savoir.

G. Vergnaud, (1987), (1996), conçoit la notion de schème comme une forme organisée et stabilisée, de l'activité de l'enseignement pour une certaine variété de situation qui appartient à une même classe et $Y$. Chevallard (1999) fait allusion à la praxéologie liée à un type de tâche d'enseignement et avance le postulat de base de la TAD (théorie de l'action didactique) où l'1on admet que toute activité humaine régulièrement accomplie peut-être subsumée sous un modèle unique.

Quant à D. Bucheton et Y. Soulé (2009), le geste professionnel concerne une certaine culture de pratique qui s'adapte au contexte : il est partagé entre professeur et apprenants, il a un but spécifique qui est de faire apprendre et éduquer, il recourt aux canaux (oral, écrit, geste), il s'adapte au milieu, il s'inscrit dans les gestes des postures pour réaliser l'efficacité.

La deuxième approche est dite phénoménologique et éthique des gestes professionnels a servi à A. Jorro, (2002) pour l'agir enseignant dans le cadre de ses recherches où elle considère le corps comme étant dans l'agir social. Le geste est, selon elle qui a été la première à introduire la notion, est un mouvement du corps adressé (porteur de valeurs), inscrit dans une situation, irrigué par la biographie et l'expérience du sujet, ayant un effet performatif auprès des élèves.

\section{MÉTHODE}

Notre travail sera focalisé sur l'approche comparative en nous appuyant sur le fondement théorique de C. Peyron-Bonjan (2010), de F. Davin et D. Groux (2009) et M.L. Schubaeur-Léoni (2009). Il s'agira pour nous non seulement de définir cette approche, mais aussi d'en présenter l'intérêt par rapport à notre recherche et servira pour analyser les gestes professionnels, des enseignants du FLE du groupe A et du groupe B.

Cette approche présente un intérêt dans ce sens qu'elle permet la réalisation des transferts des notions et concepts. Ainsi, pour notre cas l'approche comparative facilitera le 
transfert des notions auxquelles nous allons recourir pour notre analyse tels que : les gestes professionnels, l'action conjointe et le triplet de genèse comme, moyen d'amélioration des compétences de la part des enseignants. Il y a donc une nécessité de réinvestir ces termes dans le champ de la didactique du français pour mieux définir les critères d'analyse sur la base desquels la comparaison est faite. En fait, l'analyse des gestes professionnels, des enseignants du FLE, peut-être mieux perçue dans la mesure où elle est réalisée à partir de l'action conjointe c'est-à-dire : "l'action de l'enseignant et des étudiants" Forest \& A. Mercier, 2009, p.7). C'est ainsi que l'action de l'enseignant n'a du sens qu'à partir du moment où l'étudiant trouve aussi sa part même si elle insignifiante.

Pour notre travail, nous avons opté pour la méthode d'analyse basée dans l'approche clinique/expérimentale de la didactique (M.L. Schubauer-Léoni \& F. Leutenegger, 2000). Loin de nous, l'idée d'étudier cliniquement les enseignants et les étudiants, mais plutôt de voir dans quelle mesure nous pouvons élaborer une clinique des systèmes (id. p.218.).

Dans cette recherche, nous allons nous limiter à un échantillon de 4 professeurs (P1s1, P2s1, P3s1, P4s1) pour 4 classes vu le nombre limité d'enseignant du FLE, 2 universités (appelé groupe $\mathrm{A}$ et groupe $\mathrm{B}$ ). Ainsi, chaque séance correspond à une vidéo d'une heure.

Nos données sont essentiellement orales et nous les avons transcrites en vue d'analyse qualitative. L'analyse qualitative : $C^{\prime}$ est la recherche qui produit et analyse des données descriptives, telles que les paroles écrites ou dites et le comportement observatoire des personnes (S.J. Taylor et R. Bogdan, 1984). Cette analyse ne néglige pas les données quantifiables, mais ne leur accorde pas une place de choix.

Les données qui concernent notre recherche sont essentiellement qualitatives mettent l'accent sur la collecte de données principalement verbales plutôt que des données qui peuvent être mesurées. Pour notre cas nous avons utilisé les vidéos. Et quant aux données quantitatives, elles sont fondées sur l'utilisation du chiffre, mais issues des données qualitatives.

\section{RÉSULTATS ET DISCUSSION}

La grille qui fait l'objet de notre recherche vise à déterminer les difficultés de plusieurs ordres qui se dessinent devant le professeur tout comme devant l'étudiant durant l'activité. Certaines erreurs des professeurs pourront nous aider à proposer des pistes pour l'amélioration du travail. Il est sans oublier que notre recherche porte sur l'approche comparative qui vise la spécificité vers le générique en se basant sur la méthode clinique/expérimentale afin de pouvoir expliquer les différents phénomènes didactiques (M.L. Schubauer-Léoni \& F. Leutenegeger, 2002), par l'analyse de l'étudiant comme instance du système didactique.

Notre analyse didactique se base sur ces éléments suivants : l'action conjointe de G. Sensevy \& A. Mercier, 207, G. Sensevy, 2011, le triplet de genèse avec Chevallard, 1997 ainsi que les 5 préoccupations d'enchâssements de D. Bucheton, 1999; D. Bucheton \& Y. Soulé, 2009.

Ainsi, le modèle «Multi agenda des Gestes professionnels» auquel nous allons recourir, permet selon D. Bucheton \& Y. Soulé (2009) de sortir des approches élémentaires et fréquemment dichotomiques telles que pédagogie/didactique, élève/enseignant, théorie/pratique... Systémique et dynamique il met en avant cinq préoccupations centrales (qui) constituent la matrice de l'activité de l'enseignant dans sa classe, ses organisateurs pragmatiques dominants (Pastré, Mayen \& Vergnaud 2006) :

1. piloter et organiser l'avancée de la leçon : réguler le temps, l'espace, le déroulement des tâches et dispositifs d'enseignement ;

2. maintenir un espace de travail et de collaboration langagière et cognitive: réguler les relations, maintenir la cohésion du groupe, créer un climat d'apprentissage ;

3. tisser le sens de ce qui se passe : amener les élèves à faire des liens entre les tâches, avec l'avant et l'après de la leçon, le dedans et le dehors de la classe ; 
4. étayer le travail en cours : sont des gestes par lesquels l'enseignant apporte de l'aide pour une tâche que l'élève ne peut pas faire seul ;

5. tout cela avec pour cible un apprentissage, de quelque nature qu'il soit (Savoirs, techniques : cela veut dire que les quatre premières préoccupations visent l'acquisition des connaissances ou des compétences.)

Voici le tableau qui présente le code des professeurs (P1s1, P2s1, P3s1, P4s1), la leçon du jour, les différents gestes professionnels des professeurs, le pourcentage de posture ainsi que les différentes postures des étudiants qui seront analyser dans cette partie.

Tableau 1. Les gestes professionnels des professeurs ( p1s1, p2s1, p3s1, p4s1 et les postures des étudiants du groupe A et du groupe B)

\begin{tabular}{llllll}
\hline Discipline & $\begin{array}{l}\text { Code } \\
\text { professeur }\end{array}$ & Leçon & $\begin{array}{l}\text { Posture professionnelle du } \\
\text { professeur }\end{array}$ & $\%$ & $\begin{array}{l}\text { Posture des } \\
\text { étudiants }\end{array}$ \\
\hline P1s1 & Expression : jeux & Accompagnement, (1-16, 24- & $36 \%$, & Réflexive, \\
& de puzzle & 107 & $57 \%$, & $\begin{array}{l}\text { Créative, } \\
\text { première, }\end{array}$ \\
& & Contrôle, (17-23, 129-281), & & Variée
\end{tabular}

\begin{tabular}{lllll}
\hline P1s2 & $\begin{array}{l}\text { Orthographe : } \\
\text { verbe être et avoir }\end{array}$ & $\begin{array}{l}\text { Accompagnement, (L107- } \\
\text { 308, 496-498) } \\
\text { Lâcher prise (L309-495) }\end{array}$ & $\begin{array}{l}62 \%, \\
38 \%\end{array}$ & $\begin{array}{l}\text { Réflexive, } \\
\text { Variée }\end{array}$ \\
\hline P3s1 & $\begin{array}{l}\text { Expression orale : } \\
\text { analyse d'une } \\
\text { fiche }\end{array}$ & $\begin{array}{l}\text { Accompagnement (L1-295) } \\
\text { Lâcher-prise (296-472) }\end{array}$ & $62,5 \%$, & $\begin{array}{l}\text { Réflexive, } \\
\end{array}$ \\
\hline P4s1 & Expression orale & $\begin{array}{l}\text { Accompagnement (L1-24, } \\
\text { 50-169) }\end{array}$ & Variée, \\
& & $35 \%$, & Première, \\
& & & Réflexive, \\
& & & \\
\hline
\end{tabular}

En fait, cette analyse nous permettra de comparer :

L'action conjointe G. Sensery, (2007) et les cinq préoccupations du multi-agenda de Bucheton E Soulé (2009) entre les professeurs tout comme les étudiants du groupe A et ceux $d u$ groupe $B$ sur leur posture professionnelle et posture d'apprentissages selon le contexte.

Par tissage, il faut entendre l'activité du maître ou des élèves pour mettre en relation le dehors et le dedans de la classe, la tâche en cours avec celle qui précède ou qui suit, le début avec la fin de la leçon D. Bucheton \& Y. Soulé (2009). Nous pourrons aussi dire qu'il s'agit de ce qu'on a appris, ce qu'on cherche à comprendre, à faire pourquoi, comment); activité des jeux de rôle; de simulations, de compréhension écrite ou orale...

Les travaux sur le CP L.M. Brunet A. Liria, D. Bucheton (2004-2005) montrent que les bons élèves tissent eux-mêmes les liens laissés à l'état implicite par l'enseignant.
Dans la première séance du P1 [désormais P1s1], commence par rappeler aux étudiants qu'ils avaient déjà vu les jours de la semaine, les 12 mois de l'année. Et demande à l'un d'entre eux de mettre la date complète au tableau.

Après cela, le professeur demande aux étudiants de prendre leurs photocopies du livre Forum à la page 71 et se mettre en groupe de quatre.

Le P1 demande aux étudiants la signification du mot «puzzle». Personne ne répond. Le professeur insiste. L'Et1 : répond [Paz] qui signifie paix en portugais. Voyant que personne ne répondait, il répond luimême.

Exemple [1] :

P1 :[choisit quelqu'un] madame + vous comprenez le mot puzzle + a palavra puzzle + que signifie puzzle

Et2 : e paz

$\mathrm{P} 1$ : non + a palavra + puzzle + c'est un mot anglais + + donc c'est un jeu + quebra cabeça 
+ hein + é o um jogo + casse-tête ++ puzzle

$++$

Et2 : e uma palavrainglês $+c^{\prime}$ est un mot anglais [L18-23, annexe 8, P1s1, O\&E]

À travers son tissage P1 fait évoluer sa leçon à partir de la langue maternelle pour faciliter la compréhension.

Exemple [2] :

P1 : ok + elle a quel âge [reprends le P1] +

Et4 : elle a vingt-neuf ans d'âge +

P1 : maintenant je demande à mademoiselle + toi tu as quel âge +

Et5 : j'ai + vinte e um [en portugais]

P1 : tu as vingt et un d'âge + ok $+\left[s^{\prime}\right.$ adressant à un autre étudiant] +elle a quel âge

Et6 : [silence] (...)

P1 : maintenant + alors si je demande à toute la salle + vous avez un professeur de français + vous

Certains : non +

P1 : et moi + je ne suis pas votre professeur de français +

La salle : [rire]

Un étudiant : oui +

P1 : alors je vous demande la même question + vous avez un professeur de français +

La salle : oui [L25-46, Annexe 10 P1s2].

Le professeur aborde le sujet d'une manière simple afin de mettre l'étudiant en confiance. Après avoir demandé à l'étudiant, qui avait répondu vingt-neuf, P1 demande à un autre de dire l'âge de la première personne. Elle a quel âge?

En fait, P1 mène son jeu didactique afin que chacun trouve son compte dans la salle en participant d'une manière active.

La première séance du P2s1 porte sur l'accord des adjectifs numéraux cardinaux. Nous nous sommes rendu compte que la professeure avait des difficultés pour démarrer son cours. Elle accumule une série des questions mal formulées ce qui empêche un bon tissage. Mais elle a pu se rattraper peu après en demandant aux étudiants d'ouvrir leur note des cours. Ce qui permettra à l'Et6 de donner une bonne réponse : dix classes.

L'enseignante lit alors: nous avons dix classes divisées en deux groupes; les variables et les invariables. Elle demande de citer les variables d'abord et les étudiants s'exécutent.

Exemple [3] :

P2 : il y a combien des classes des mots +
: réponds dans la masse + les adjectifs cardinaux.

: non + il y a combien des classes des mots + il y a en a combien des classes des noms $+[\ldots]$

Et6 : dix classes +

P2 : tu as 1 point + tu as 1 point + [en pointant l'étudiant du doigt] + nous avons dix classes des mots + diviser en deux parties + les classes des variables et la classe des invariables + ce n'est ça +

[L18-21, 34-37, annexe 11, P2s1]

Il s'agit de l'interprétation d'un horaire de classe (document authentique) à l'école secondaire. L'enseignant donne un exemple d'interprétation de l'horaire avant de choisir différents étudiants pour le faire chacun avec ses mots. Il s'agit d'un bel exercice de communication. La classe est motivée, l'enseignant accorde la parole à tous les étudiants, même ceux qui n'avaient pas le courage de parler par peur de se tromper.

Exemple (4) :

P3 : oui+ mathématique +

Et32 : le professeur de mathématique + il est cours (sic) + rire + je vous coûte (dit le P3) $+2 \mathrm{~h}$ à $10 \mathrm{~h}$ il est cours (sic) + les premiers temps + de $2^{\circ}$ à $18 \mathrm{~h}+$ il est \# (sic)

P3 : écoutez encore un autre point + une autre consigne + la classe + vous agissez + vous réagissez lorsque l'autre a commis d'erreurs d'accord $+d^{\prime}$ accord $+(\ldots)$

Et 33 : le jeudi professeur +

P3 : oui + le jeudi + s'il vous plaît mathématique +

Et33 : le professeur de mathématique + il a cours à $13 \mathrm{~h}$ à $14 \mathrm{~h}$ et de $14 \mathrm{~h}$ à $15 \mathrm{~h}$ en $6^{\circ}$ année $++3^{\circ}$ année pardon + (intervention) + il a dit + il y a cours + (L275-297, annexe 14, P3s1)

Il s'agit d'une leçon d'expression orale et écrite portant sur trois petits dialogues. Il sera question de lire et de jouer le rôle deux à deux. Le tissage du $\mathrm{P} 4$ réside dans le simple fait de répéter le dialogue.

Exemple (5) :

P4 : c'est clair + nous allons passer à un autre dialogue + bonjour + madame + bonjour + monsieur + excusez-moi + vous vous appelez comment + Corinne LAPORTE + et vous + Gérard LEROY + L+ E + R + O + $\mathrm{Y}+$ je répète bonjour madame (...) (il choisit deux étudiants pour faire le dialogue) 
(L71-78, annexe15, P4s1).

Nous avons relevé que d'une manière générale que les professeurs du groupe $\mathrm{A}$ tissent plus à partir de la langue maternelle pour faire avancer le savoir tandis que ceux du groupe $B$ le font en français.

L'Atmosphère, nous dit D. Bucheton \& Y. Soulé, 2009, c'est l'espace intersubjectif qui organise les différentes rencontres (intellectuelle, relationnelle, affective, sociale) entre des individus qui ont en commun les mêmes préoccupations. C'est cet éthos D. Maingueneau (1997) qui est le lieu dans lequel baignent les interactions et qui en même temps les colorent d'une certaine tonalité : sérieuse, ludique, tendue, ennuyeuse, voire inquiétante.

P1 a créé un climat de travail abordable, même s'il s'agit de la traduction au lieu des questions de réflexion ou de favoriser plus les interactions langagières. Cette attitude a permis d'améliorer l'atmosphère en entraînant une participation remarquable de la classe. L'enseignant a su bien gérer le temps et l'espace : circule, parle, observe, contrôle, s'assied, reprend le mouvement, etc.

Exemple (6) :

Paella : c'est une spécialité espagnole ; paella é um prato espagnol (en portugais pour mieux se faire comprendre.

Sushi : c'est un plat chinois [sic] à base de poisson cru [peixe cru] en portugais); Couscous : tipo funje feito com a farinha (en portugais).

Cet exemple nous montre le déplacement du professeur dans la salle.

Exemple (7) :

P1 : (commence à mettre le tableau pour être complété après) + + jeu de puzzle Forum page $71 / / / l^{\prime}$ Argentin + il mange + il boit + il arrive (il remplit le tableau sans rien dire et après circule dans la salle) + ici vous avez fini +ça marche ++ (il circule à travers les différents groupes)///(il s'arrête de temps à autre et parle avec les étudiants)///(et corrige certaines fautes d'orthographe) exemple+ l'article partitif ok + (un étudiant appelle le P1 un problème) + (une étudiante se + met à parler avec le professeur) $+^{* * *}$ (L108-115, annexe8, P1s1).

Durant la première séance du P2, l'atmosphère n'était pas du tout abordable à la suite de la mauvaise formulation des questions et de la traduction littérale au lieu d'une traduction littéraire. En plus, la professeure ne circulait pas tellement, elle était plus devant le tableau ou assise. Cette distance n'a pas aussi facilité la tâche.

Exemple (8) :

P2 : nous avions dit que les adjectifs numéraux cardinaux $+\mathrm{c}^{\prime}$ est quoi + (sic) $\mathrm{c}^{\prime}$ est + (elle change la question) + d'abord d'où viennent les adjectifs numéraux cardinaux + (sic) + dans quel chapitre + les adjectifs numéraux cardinaux viennent de quelle classe +

Et2 : dans la classe des mots $+\mathrm{j}$

P2 : il y a combien des classes des mots +

Et3 : réponds dans la masse + les adjectifs cardinaux.

P2 : non + il y a combien des classes des mots + il y a eu combien des classes des noms + (L13-21, annexe11, P2s1).

La traduction est faite lorsqu'il y a blocage dans la compréhension, mais P2 s'est créée des problèmes inutilement. Elle voulait tout traduire (du français en portugais). Cette situation a créé autant de murmures dans la salle.

À travers sa leçon, le professeur donne la possibilité aux étudiants de se sentir à l'aise dans la classe malgré les erreurs et tient à ce que les étudiants ne se moquent pas entre eux, pour décourager ceux qui ont des problèmes d'expressions. Il se déplace dans toute la salle, se rapprochant de plus en plus des étudiants afin de briser la barrière de distance qui le sépare, intervient à peine, contrôle et applique l'effet maître selon D. Bucheton (2014) qui sont: relations, engagement, éthique, langages, espace pour penser.

Exemple (9) :

P3 : Pedro + tu n'as pas suivi + Pedro + il fallait dire quoi + + oui + Pedro +

Et45 : hum +

P3 : mercredi + mathématique +

Et45 : hum + hum + (il tousse et fait signe d'avoir mal à la gorge) +

P3 : (choisis une autre personne) $+($ L422-426) (annexe13, P3s1)

Dans sa séance, malgré le recours fréquent $\mathrm{du}$ professeur à la répétition, la classe est motivée et participe à l'activité. L'enseignant circule normalement dans la 
classe, se rapprochant de plus en plus des étudiants, créant ainsi une atmosphère de confiance pour le travail qui était basé seulement sur la répétition. Il a su bien gérer le temps et l'espace.

Exemple (10) :

P4 : tu es capable ou pas + tu es capable ++ excusez-moi (commence le professeur)

Et10 : excusez-moi monsieur vous êtes de l'Union européenne+

Et9 : non + je suis brésilien

Et10 : alors + remplissez cette fiche s'il vous plaît+

Et9 : merci

P4 : ok + très bien + deux autres + allez du courage + allez lève-toi + on y va + allez (L239-244, annexe16, P4s2).

Cet extrait nous montre le climat du travail. Le professeur encourage même ceux qui ont du mal à parler. Cela a fait que tous les étudiants se sont sentis en confiance et ont participé massivement à l'activité.

Nous avons constaté que les professeurs du groupe A avaient assez bien géré la salle, mais ne circulaient presque pas en dehors du P1 qui circulait, contrôlait et était plus proche des étudiants; en revanche, ceux du groupe B ont bien géré la classe, circulaient, contrôlaient, encourageaient, voire lançaient quelques blagues et étaient plus proches des étudiants.

Parlant du savoir, nous faisons allusion au savoir-être, savoir-faire... La visée peut en être floue, d'autant que le rapport au savoir et son évolution comportent une dimension socio-affective et expérientielle très importante.

L'écrit, contrairement aux méthodes traditionnelles et audiovisuelles, devient un acte de communication fonctionnel, un savoir et savoir-faire spécifique permettant à l'apprenant de s'exprimer et de communiquer au moyen de signes spécifiques, les signes graphiques.

L'enseignant commence par placer quelques éléments de l'exercice dans le tableau pendant que les étudiants travaillent d'abord en groupe. Après le travail en groupe, P1 demande à chaque représentant du groupe de passer devant pour remplir le tableau par les éléments qui manquent et de justifier aussi la réponse.

Exemple (11) :

P1 : pourquoi + qu'est-ce que vous avez fait pour trouver + arriver à cette réponse +

Et21 : numéro 6 (il lit) + le Japonais n'arrive pas au restaurant à une heure et il boit de la bière +

P1 : il y a une information précise +

Tous : oui +

P1 : donc + le Japonais+ donc + remplissons déjà + le japonais boit de la bière ok + et à quelle heure il est arrivé au restaurant +

(L241-248, annexe8, P1s1).

Les étudiants remplissent le tableau tout en justifiant le pourquoi de la réponse ce qui démontre une certaine maîtrise de leur part.

La leçon du jour porte sur l'accord de quelques adjectifs numéraux cardinaux. Durant la leçon elle explique à partir des exemples des étudiants les différents accords. Or, elle aurait pu à partir des exemples faire comprendre le fonctionnement de la règle ce qui faciliterait la compréhension.

\section{Exemple (12) :}

P2 : la salle est-ce que c'est cent et un +++ dizemos que «et » avec le trait d'union il n'y a que dans les dizaines + unité + il n'y a que dans le vingt à partir de + eh + + dixsept + dix-huit traits d'union + dix-neuf trait d'union + est-ce que c'est multiplié + dix-huit + vingt sozinho $+(\ldots)$. il y a des exceptions + il y a des lois qu'il faut suivre++ dans le cent et un + humm + até cem não ha « $e$ " não ha traço + não ha nada +il n'y a rien + (elle efface-le « et» entre cent +un) ce qui donne +cent un + dans dix (L415-434, annexe 11, P2s1).

En effet, nous avions eu l'impression que le savoir n'était pas assez bien défini, raison pour laquelle il y a eu peut-être des problèmes de tissage et surtout d'atmosphère de la classe.

P3 recourt au portugais dans le cas d'appui pour faciliter la compréhension. Par exemple lorsqu'il corrige (il est, il a) on peut penser que la question il est/il a c'est une question (syntaxique/phonologique) et là bizarrement le P3 ne soulève pas le problème.

Le constat général est que les étudiants parlent et discutent. Bonne utilisation du FLE. Il arrive que le professeur soit plus sur le plan de la 
normalisation, lorsqu'il corrige l'Et32 qui confondait il est cours/il a cours.

Exemple (13) :

P3 : continua + continua repetir ++ on y va ici ++++ vous avez deux minutes + nous allons préparer la première partie + depois vamos + hein + la première partie + au prochain tour va introduire la dernière partie + na proxima fez vamos introduzir a ultima preoccupação +

(L31-345, annexe14, P3s1).

À travers l'exercice le professeur fait circuler le savoir acquis entre les étudiants afin de mieux retenir et appliquer dans le contexte.

P4 accorde aux étudiants le temps de s'exprimer devant la salle, après s'être préparé deux à deux. Ici, il ne s'agit pas du tout de la communication selon l'approche communicative, mais plutôt d'une répétition à partir des exemples ou mieux encore du modèle du P4.

Exemple (14) :

P4 : maintenant pour terminer vous venez devant pour présenter le premier dialogue + (L70-71, annxe15, P4s1)

Tous les professeurs ont essayé de bien gérer l'objet du savoir durant leur séance, car la cible visée par la situation d'enseignement/l'apprentissage était connue.

Mais c'est seulement l'application des concepts, des techniques, des attitudes, des méthodes, des stratégies, des comportements scolaires, sociaux, des pratiques sociales, des formes d'adaptation et d'ajustement aux situations, etc., qui changent d'un professeur à l'autre.

À ce niveau nous remarquons que les professeurs du groupe A ont eu un problème dans la transmission du savoir (notamment le cas du P2) par rapport à leurs congénères du groupe B qui s'en sont mieux sortis.

Le pilotage a pour but d'organiser la cohérence et la cohésion de la séance, d'assurer la chronogenèse de la leçon. A cet effet, après avoir donné pour consignes de se mettre en groupe de 4 ou 5, le professeur procède à la lecture de l'exercice qui porte sur le jeu de puzzle. Il explique les mots difficiles et la manière dont les étudiants doivent travailler. À la fin, les étudiants font ce que le professeur attend d'eux : le remplissage de la grille au tableau à tour de rôle par le délégué de chaque groupe.

\section{Exemple (15) :}

P1 : + rempli (sic) ce tableau + remplissez le tableau et se basant sur ces informations ok $+1+2+3+4+6+$ ok + donc $+c^{\prime}$ est un jeu de puzzle + le quebra cabeça não hein + à partir des informations que vocês tenham + não hein +então vão sabercompletar ou + preencher /// travailler en groupe + discuter pendant ce temps moi $+(\ldots)$ je vous en prie + il ne faudra pas remplir sur le livre (sic) + procuram uma folha + alguém vai iniciar a escrever etrabalhar + trabalhar + il ne faudrait pas remplir sur le livre +ok///(il se retourne encore vers les étudiants) + est-ce que vous avez compris + (L95-104, annexe8, P1s1).

Le travail en groupe permet aux uns et aux autres de s'exprimer facilement; mais le fait est qu'ils s'expriment en portugais et écrivent en français. Ce qui est déjà positif.

L'enseignante fait usage des stratégies en demandant de conjuguer le verbe travailler au passé composé. Après la réponse $\mathrm{P} 2$ gratifie l'Et10 pour le motiver davantage. Cette attitude a fait que les étudiants ont commencé à travailler.

\section{Exemple (16) :}

P2 : tu as 1 point plus le 0,5 de la fois passée

$$
++
$$

Et14 : merci

P12 : je vais te donner encore 1 point

Et14 : merci madame

P2 : qui encore + quem ++ (silence) le problème qui est là est que nous (...) (L207-211, annexe13, P2s1)

P3 favorise le déblocage des étudiants par le fait qu'il est en communication permanente avec eux; il crée des situations de dialogues, laisse les étudiants parler; il créer un environnement (une ambiance) francophone favorable pour l'utilisation de la langue.

Exemple (17) :

P3 : écoutez encore un autre point + une autre consigne + la classe + vous agissez + vous réagissez lorsque l'autre a commis d'erreurs + d'accord + d'accord + (L296298, annexe13, P3s1)

Pour le P4 à travers sa séance, recourt à plusieurs stratégies : celle de faire parler tout le monde même ceux qui ont des problèmes 
de lecture ou de prononciation, en leur faisant répéter et surtout en les encourageant par les acclamations.

\section{Exemple (18) :}

P4 : tu es capable ou pas + tu es capable ++ excusez-moi (commence le professeur)

Et10 : excusez-moi monsieur vous êtes de l'Union européenne+

Et9 : non + je suis brésilien

Et10 : alors + remplissez cette fiche s'il vous plaît+

Et9 : merci

P4 : ok + très bien + deux autres + allez du courage + allez lève-toi + on y va

+ allez

Et11 : excusez-moi monsieur vous êtes de l'Union européenne+

(L239-245, annexe16, P4s1).

Les professeurs de deux universités ont tous eu recours aux stratégies et consignes différentes qui leur ont permis de bien gérer leur classe à des niveaux différents. Ceux du groupe A ont eu certaines difficultés à certains moments, mais ils ont compris qu'il fallait changer des stratégies au fur et à mesure qu'évoluer la séance, tel le cas du P2.

Mais ceux du groupe B semblent mieux s'en sortir du début à la fin de l'activité à travers les consignes, les stratégies mises en place au cours de l'évolution de chaque séance.

Notre analyse des postures enseignantes a de l'influence sur les étudiants et leur gestion de l'avancée du savoir, raison pour laquelle nous avons tenu à les traiter ensemble.

Sur ce, les postures professionnelles adoptées par l'enseignant pendant la séance tiendront compte, de plusieurs facteurs et peuvent varier d'un moment à l'autre.

Le concept de posture professionnelle prend sa source dans la théorie des concepts en acte de G. Vergnaud (1996). La définition donnée par D. Bucheton et Y. Soulé (2009) définissent la posture comme :

"Un schème préconstruit $d u$ "penser-direfaire" que le sujet convoque en réponse à une situation ou à une tâche scolaire donnée. La posture est relative à la tâche, mais construite dans l'histoire sociale, personnelle et scolaire du sujet » (p. 38).

Nous rappelons que la notion de posture est comparable à celle de la topogenèse G. Sensevy \& A. Mercier, (2007) et que sur les cinq postures existantes d'apprentissage des étudiants, seuls trois apparaissent tout au long des séances ou activités de classe. Il s'agit de la posture: réflexive ou seconde, ludique ou créative, et première.

Ainsi, le tableau qui suit contiendra les gestes professionnels des professeurs et les postures des étudiants, car l'action du professeur est orientée en fonction des conduites potentielles et effectives de ses étudiants d'une part, et l'action de l'étudiant est en partie contrainte par les choix de l'enseignant.

Tableau 2. les gestes professionnels des professeurs et les postures des étudiants du groupe A et B

\begin{tabular}{|c|c|c|c|c|c|}
\hline Discipline & $\begin{array}{l}\text { Code } \\
\text { professeur }\end{array}$ & Leçon & $\begin{array}{l}\text { Posture professionnelle du } \\
\text { professeur }\end{array}$ & $\%$ & $\begin{array}{l}\text { Posture des } \\
\text { étudiants }\end{array}$ \\
\hline & P1s1 & $\begin{array}{l}\text { Expression: jeux de } \\
\text { puzzle }\end{array}$ & $\begin{array}{l}\text { Accompagnement, } \quad(1-16, \\
24-107) \\
\text { Contrôle, }(17-23,129-281), \\
\text { Lâcher-prise, (189-128) }\end{array}$ & $\begin{array}{l}36 \%, \\
57 \%, \\
7 \%\end{array}$ & $\begin{array}{l}\text { Réflexive, } \\
\text { Créative, } \\
\text { première, } \\
\text { Variée } \\
\end{array}$ \\
\hline & P2s1 & $\begin{array}{ll}\text { Grammaire : } & \text { l'accord } \\
\text { des } & \text { adjectifs } \\
\text { numéraux cardinaux }\end{array}$ & Contrôle & $100 \%$ & Première \\
\hline & P3s1 & $\begin{array}{l}\text { Expression orale: } \\
\text { analyse d'une fiche }\end{array}$ & $\begin{array}{l}\text { Accompagnement (L1-295) } \\
\text { Lâcher-prise (296-472) }\end{array}$ & $\begin{array}{l}62,5 \% \\
37,5 \%\end{array}$ & $\begin{array}{l}\text { Réflexive, } \\
\text { Variée, }\end{array}$ \\
\hline & P4s1 & Expression orale & $\begin{array}{l}\text { Accompagnement (L1-24, } \\
\text { 50-169) } \\
\text { Contrôle (L25-49, 170-223) }\end{array}$ & $\begin{array}{l}65 \%, \\
35 \%\end{array}$ & $\begin{array}{l}\text { Première, } \\
\text { Réflexive, }\end{array}$ \\
\hline
\end{tabular}


Ce tableau nous présente les différents gestes et postures auxquels les professeurs et les étudiants ont recouru durant les différentes activités. Ces postures sont mises en pourcentage à partir du notre corpus constitué par les différentes transcriptions de toutes les séances.

Dans la première séance, la position du P1 évolue au fur et à mesure, qu'évolue la leçon. Tenant compte surtout de la réaction des étudiants. Au début de la leçon, sa posture est celle de l'accompagnement; lorsqu'il se rend compte que les étudiants sont en difficulté, il passe à la posture d'enseignement en se faisant maître dans la salle peut-être pour ne pas faire passer le temps pour rien. Il évolue enfin en posture de lâche prise qui consiste à laisser travailler les étudiants seuls.
Cette position rend les étudiants responsables et les motive de plus en plus de se prendre en charge.

\section{Exemple (19) :}

P1 : vous allez remplir le tableau + donc + là vous allez remplir + nous avons + l'Italien + le Japonais +et le Marocain///alors vous allez dire l'Argentin - ce qu'il a mangé + hein + ok + la $1^{\circ}$ colonne vous avez $+\mathrm{il}$ mange $+2^{\circ}$ colonne + il boit + ok + et $3^{\circ}$ colonne + heure d'arrivée + hora de chegada + donc + rempli (sic) ce tableau + (...) à partir des informations que vocês tenham + não hein +então vão sabercompletar ou + preencher///travailler en groupe + discuter pendant ce temps moi + je passe le tableau au tableau (L90-100, annexe8, P1s1).

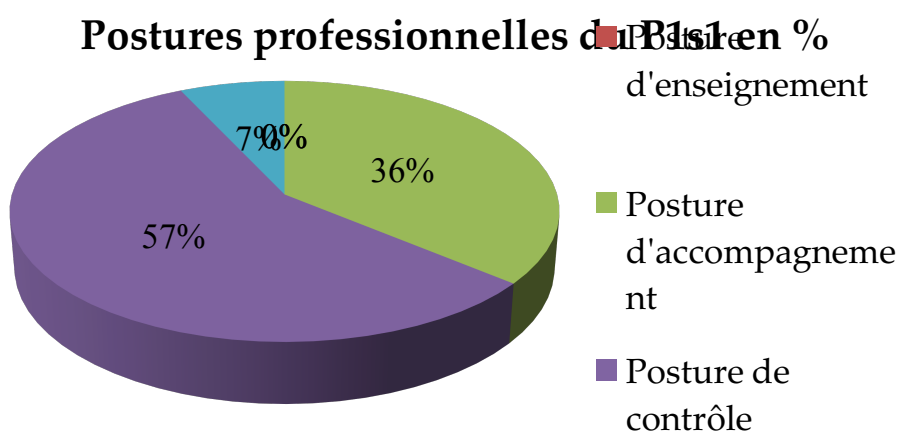

Graphique 1. postures professionnelles du P1s1

Ce graphique montre l'évolution des gestes professionnels du P1s1, que sa séance était plus axée sur le geste de contrôle, suivi de l'accompagnement et du lâcher-prise qui n'a occupé que $7 \%$ de son activité. P1 après avoir vérifié que les différents groupes avaient fini de résoudre leur exercice, il choisit un membre par groupe pour répondre en complétant la grille au tableau.

Exemple (20) :

P1 : il montre du doigt la phrase au tableau + L'Argentin + qu'est-ce qu'il mange

Et7 : de la paella +

P1 : oui + mais comment est-ce que vous avez trouvé cette réponse + qu'est-ce que vous avez fait pour affirmer que l'Argentin mange de la paella $+(\ldots)$

Tous : du vin

P1 : donc + nous avons une information précise $+l^{\prime}$ Argentin arrive au restaurant une heure et demie et il mange une spécialité espagnole + donc c'est correct + nous avons donc $+\mathrm{l}^{\prime}$ Argentin mange +

Tous : de la paella +

P1 : et qu'est-ce qu'il boit +

Tous : du vin (L137-153, annexe8, P1s1).

Les étudiants à travers leur délégué de groupe défilent à tour de rôle au tableau pour remplir la grille. L'enseignant n'intervient qu'en cas de nécessité pour orienter vers une bonne réponse. (Posture d'accompagnement et de lâcher-prise).

Les gestes du P2 à travers la séance, nous fait voir qu'elle est le plus souvent dans une posture de contrôle des étudiants, de contrôle de l'avancée de la tâche. Durant la leçon sur les accords des adjectifs, son souci premier semble être le pilotage visant à arriver très vite vers l'épisode suivant. Du 
coup, la parole de certains élèves est confisquée, et l'atmosphère est contrainte.

Postures professionnelles du P2 1 en

$\%$

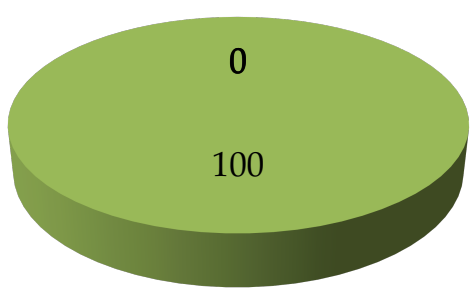

- Posture

d'enseignement

Posture

d'accopagneme

nt

Graphique 2. postures professionnelles du P2s1

Cette figure nous montre que la professeure est restée durant la séance au niveau de la posture de contrôle. Elle interrogeait pour avoir des réponses précises des étudiants et ne les laissait pas réfléchir seul.
La posture d'accompagnement domine dans la première séance du P3. Mais elle était secondée de temps à autre par celle du lâcherprise comme nous montre le graphique cidessous.

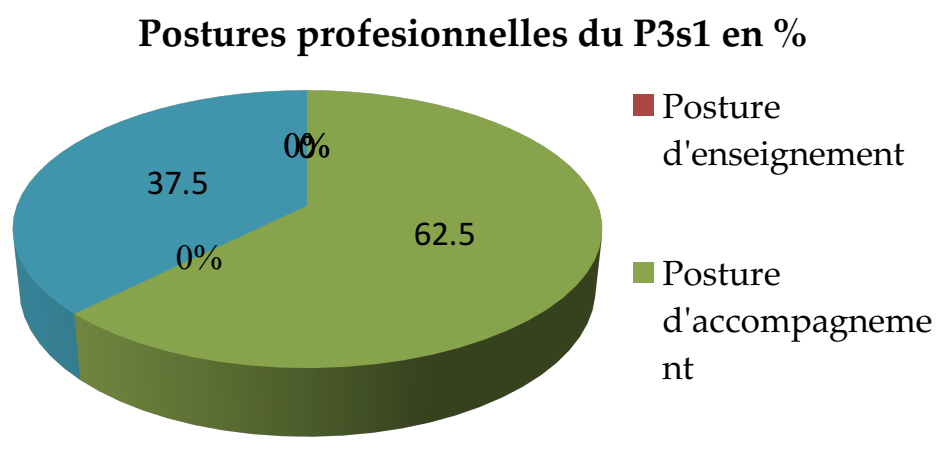

Graphique 3. postures professionnelles du P3s1

L'exemple qui suit nous présente, une situation de l'utilisation de la posture d'accompagnement par le professeur. Il s'agit en fait d'un échange entre le professeur et l'Et7 sur l'analyse d'une fiche d'horaire de cours au secondaire.

\section{Exemple (21) :}

P3 : une autre version + qui peut procéder $\mathrm{d}^{\prime}$ autre manière + traduire d'une autre façon + alors voyons alors lundi de sciences physiques $+(\ldots)+$ qu'est-ce que vous avez dit tantôt (s'adressant à un étudiant) + alalala + ) + il choisit une autre personne)

Et7 : le professeur de +

P3 : de physique +

Et7 : le professeur de physique de $18 \mathrm{~h}$ à $20 \mathrm{~h}+$ mais il a cours à $13 \mathrm{~h}$ et $16 \mathrm{~h}+13 \mathrm{~h}+14 \mathrm{~h}$ il a cours +14 h 15 il est cours (sic) $+13 \mathrm{~h}$ $-4 \mathrm{~h}$ il est cours en $6^{\circ}$ classe + en $6^{\circ}$ année + le cours $4^{\circ}$ classe + (L36-44, annexe 13, P3s1).

P4 recourt plus à la posture de contrôle dans sa séance, transite par la posture d'accompagnement à travers la répétition. Exemple (22) :

P4 : choisit deux étudiants pour faire la lecture $+$

Et5 : salut + je m'appelle Carlos (ton descendant $)+$

P4 : (réagit) + non + salut (ton montant) + je m'appelle Carlos+ vas- $y^{+}$

Et 5 : (reprend) + salut+ je m'appelle Carlos + et toi tu t'appelles comment (...)

Et5 : Corinne + 
P4 :très bien + c'est clair + (L61-69, annexe15, P4s1)

Quant à l'exemple qui suit, il présente une situation de la posture d'accompagnement.
À travers le graphique ci-dessous, nous avons la présentation de la première séance du P4 qui n'a recouru qu'à deux postures : celle d'accompagnement qui est majoritaire et celle du contrôle, ignorant toutes les autres.

\section{Postures professionnelles du P4s1 en \%}

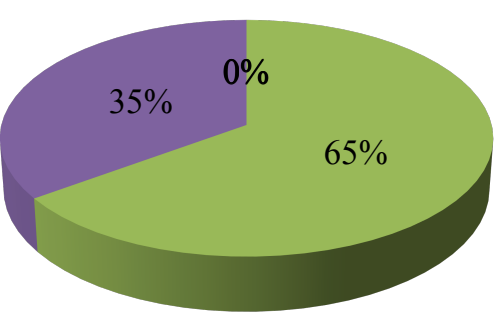

Posture

d'enseignement

Posture

d'accompagneme

nt

Posture de

contrôle

Graphique 4. postures professionnelles du P4s1

Notons que, le professeur expérimenté circule entre les différentes postures de contrôle. Les graphiques qui postures, par contre le professeur débutant est ou reste davantage dans les

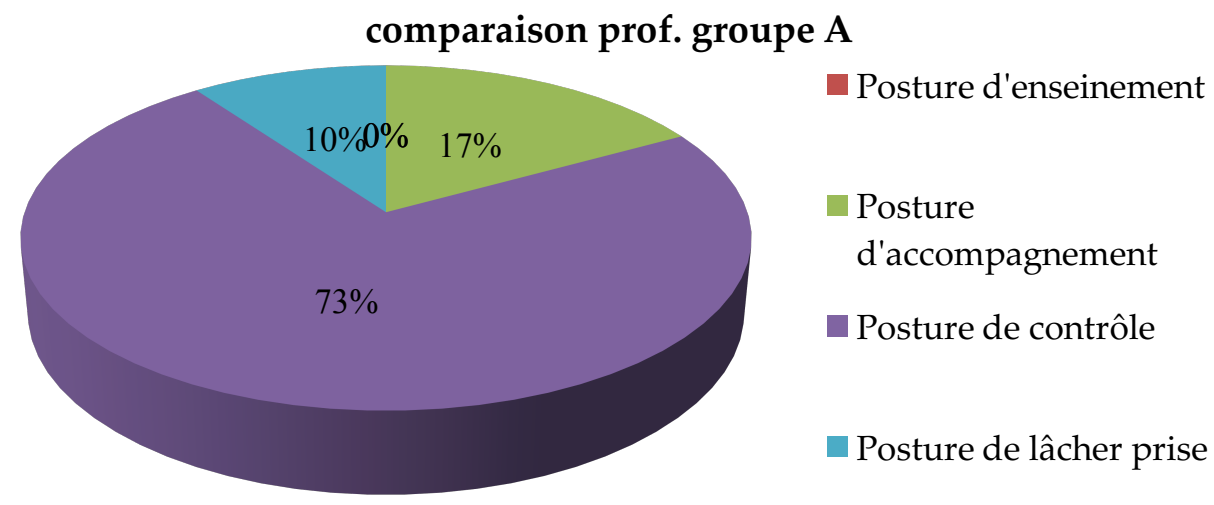

Graphique 5. comparaison prof du groupe

Ce graphique nous montre que les professeurs du groupe A évoluent plus dans la position de contrôle, suivi de la position d'accompagnement et le lâcherprise n'est qu'à $10 \%$. Ce qui revient à dire $q u$ 'il y a une faible place pour les interactions entre étudiants. En fait, les professeurs ont enseigné sans toutefois tenir compte de l'étudiant qui doit être considéré comme le centre de l'apprentissage et comme un spectateur. 


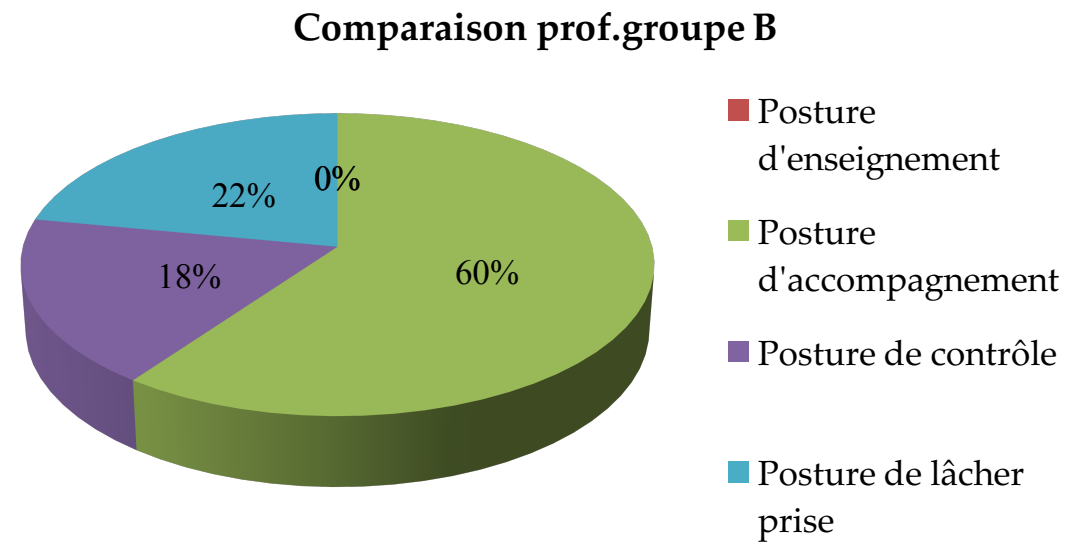

Graphique 6. comparaison prof. Groupe B

Ce graphique montre que les professeurs du groupe B sont plus dans la position $\mathrm{d}^{\prime}$ accompagnement. Ils enseignent plus en accompagnant l'étudiant; vient ensuite la position de lâcher-prise à $22 \%$, moment où les professeurs laissent les étudiants évoluer seul. Et enfin vient le tour de la posture du contrôle qui est à $18 \%$.

De ces deux graphiques, nous dirons que les enseignants du groupe B semblent mieux être dans le processus apprentissages/enseignements que ceux du groupe A.

Comme nous l'avions dit supra, les postures des professeurs influencent d'une manière ou d'une autre celles des étudiants. C'est ainsi qu'à ce niveau de comparaison pour le groupe B nous avons plus la posture première et réflexive et très peu de postures variées, et pour ceux du groupe B nous aurons plus la présence de la posture réflexive et variée et très peu de la posture première.

Le résultat obtenu à travers de la théorie de l'action conjointe et de geste professionnel nous ont permis de nous rendre compte que les professeurs du groupe A semblent aguerri par rapport à ceux du groupe B peut-être pour avoir bénéficié de la formation. Mais un échantillon plus large nous aurai peut-être donner d'autre résultat.

Nous pensons que notre analyse nous a permis de répondre à notre problématique qui concerne les difficultés des étudiants seraient en lien avec les difficultés des enseignants peu formés à enseigner le FLE.

\section{CONCLUSION}

Dans cette partie qui concerne l'analyse didactique que nous avons réalisée à partir d'une liste de critère d'analyse, nous nous sommes aperçus que les professeurs semblent avoir des difficultés pratiques dans l'utilisation de l'action conjointe, des cinq préoccupations de l'enchâssement selon D. Bucheton (tissage, l'atmosphère, le pilotage...) et de l'évaluation.

Dans la plupart des cas, le pilotage des postures professionnelles pose problème : la plupart du temps, les professeurs restent dans la position de contrôle et de l'accompagnement. Ils sont peu dans la posture de lâcher-prise. Ce qui entraîne aussi les étudiants à rester plus dans la posture réflexive et première. Ils ont peu d'opportunités d'adopter des postures variées, car les professeurs ne leur donnent pas l'occasion d'y rester longtemps.

En fait, nous dirons que les professeurs semblent avoir des problèmes liés à la méthodologie et au manque de documentation. Cette situation de manque se reflète chez les étudiants en termes de difficulté langagière.

En fonction des différents cas relevés, les enseignants semblent reconnaître leurs limites et ont même proposé de suivre la formation continue et de commencer le FLE 
dans les classes précédentes pour aider les étudiants.

\section{RÉFÉRENCES}

Bucheton, D., Dezutter, O. (Sous la direction de) (2008), Le développement des gestes professionnels dans "enseignement $d u$ français. Un défi pour la recherche et la formation: Éditions de Boeck Université. Bruxelles.

Bucheton, D. \& Soulé, Y. (2009). Les gestes professionnels et le jeu des postures de l'enseignant dans la classe : un multi-agenda de préoccupations enchâssées. Éducation et Didactique, 3(3), 29-48.

Bucheton, D. (décembre 2008). L'agir enseignant, une question d'ajustement, collectif, Bucheton: direction, édition Octares. Toulouse.

Chevallard, Y. (1999). L'analyse des pratiques enseignantes en théorie anthropologique $\mathrm{du}$ didactique. Recherches en didactique des mathématiques, 19(2), 221-266.

Conseil de l'Europe. (2001). Le Cadre européen commun de référence pour les langues. Conseil de l'Europe: Les Éditions Didier, Paris 2001

Dudois, J. (2012). Giacomo, M., Guespin, L., Marcellesi, C., Méve, J.P, Les Grands Dictionnaires LAROUSSE, le dictionnaire DE LINGUISTIQUE et des Sciences $d u$ langage. Édition, Larousse P100.

Desmons, F., Ferchaud, F., Godin, D., Guerrieri, C., Clément, S. Jourdain, S., J, Kempf, M., Lancien, F., Razakamana, R. (2005). Enseigner le FLE, Pratiques des classes. Paris: Belin

Groux, D. et Davin-Chnane F. (2009). Méthodologie de la comparaison en éducation, Raisons, comparaisons, éducations, La Revue française d'éducation comparée $\quad \mathrm{N}^{\circ} 5$ : Harmattan. Paris

Jorro, A. (2002) Professionnaliser le métier d'enseignant. Esf éditeur.

Pastré, P., Mayen, P., \&Vergnaud, G. (2006), La didactique professionnelle, Revue française de pédagogie, $\mathrm{n}^{\circ} 154$, janvierfévrier-mars 2006 145-198.

Peyron, B. (2010). Problèmes épistémologiques de la didactique comparée. Concepts, champ(s) théorique(s)... In Groux, D \& F. Chnane-Davin, F. Méthodologie de la comparaison en éducation. Raisons, Comparaisons, Education, $L a$ revue française d'éducation comparée, 5. Paris : l'Harmattan.

Sensevy, G. et Mercier, A. (2007). Agir ensemble. L'action didactique conjointe du professeur et des élèves: Presses universitaires de Rennes. Rennes

Sensevy, G., Mercier, A. et Schubauer-Léoni, M.L. (2000). Vers un modèle de l'action didactique du professeur. À propos de la Course à 20, Recherchesen Didactique des mathématiques, 20 (3), 263-304.

Schubauer-Léoni, M.L. \& Leutenegger, F. (2002) Expliquer et comprendre dans une approche Cliniquelexpérimentale $d u$ didactique d'ordinaire. In Leutenegger, F.\& Saada-Robert Edition

Schubauer-Léoni, M.-L, (2009) Les outils de la comparaison en éducation in Éducation Comparée, $\mathrm{n}^{\circ} 5$, méthode de la comparaison, l'Harmattan

Taylor, S. J., \&Bogdan, R. (1984) Introduction to qualitative research methods: The search For Meanings: John Wiley \& Sons. New York

Vergnaud, G. (1992). Approches didactiques en formation d'adultes. Éducation Permanente. III 